\section{Correlação entre Ultrassonometria Quantitativa de Calcâneo e Densitometria Óssea Duo-Energética de Coluna e Fêmur na Avaliação Óssea}

\begin{abstract}
RESUMO
A densitometria óssea com raio $X$ duo-energético (DEXA) é o método atualmente mais utilizado para medição de massa óssea; porém, a ultrassonometria óssea quantitativa (USQ) vem apresentando resultados promissores na predição de fraturas. Visando comparar DEXA e USQ, correlacionamos os resultados obtidos com estes métodos em pacientes rotineiramente encaminhados para realização de DEXA em nosso serviço. Estudamos 165 mulheres e 24 homens com idades entre 20 e 84 anos (Mi: 51 e 53 anos, para mulheres e homens respectivamente) com diagnósticos variados. Todos foram submetidos a medição da densidade mineral óssea (DMO) pela DEXA (Lunar DPX-L) em coluna lombar (LOMB) e em colo (COLO) e trocanter maior (TROC) e USQ em calcâneo (Lunar- Aquilles), onde foram considerados os parâmetros de velocidade do som (SOS) e atenuação do som (BUA). Houve correlação positiva significante entre as medidas de DMO em todos os sítios e SOS e BUA. Quando separamos por sexo, a melhor correlação foi observada nos homens, entre TROC e SOS, com $r=0,82$. Quando separamos por idade, a correlação foi menor naqueles com mais de 50 anos, comparados aos com menos de 50 anos. Dentre os 89 pacientes considerados normais pela DEXA em LOMB, 29,8\% apresentavam valores abaixo de IDP na USQ. Por outro lado, $35,8 \%$ dos indivíduos cujos valores de USQ estavam a menos de IDP abaixo da média, apresentavam-se com osteopenia ou osteoporose $(T<-1)$ pela DEXA de LOMB. O coeficiente de variação dos valores obtidos de USQ para as 9 medidas realizadas na mesma pessoa foi de $0,66 \%$ para SOS e $2,75 \%$ para BUA. Concluindo, a USQ correlaciona-se apenas moderadamente com DMO e, portanto, sua utilização como um teste de rastreamento para a realização de DEXA é de pouca utilidade, uma vez que a discordância entre os métodos para um mesmo individuo é elevada. O valor da USQ na avaliação do risco de fratura já está estabelecido, porém estudos prospectivos são necessários para que se padronize sua utilidade no diagnóstico e acompanhamento das doenças ósseas. (Arq Bras Endocrinol Metab 1999:43/3 205-209).
\end{abstract}

Unitermos: Ulitrassonometria óssea; Densitometria óssea; Massa óssea

\section{artigo original}

\author{
Marise Lazavetti Castro \\ Cynthia Brandão \\ Mirna Yae Yassuda \\ Luciana N.C. Martin \\ José Gilberto H. Vieira
}

Disciplina de Endocrinologia e Metabologia, Departamento de Medicina, Universidade Federal de São Paulo / Escola Paulista de Medicina (UNIFESP/EPM), São Paulo, SP.
Recebido em $28 / 04 / 98$ Revisado em $21 / 05 / 99$ Aceito em 27/05/99 
DEXA values in all sites and USQ. The best correlation was seen in men, between TROC and SOS $(r=0.82)$. When only individuals 50 years or older $(n=102)$ were evaluated, the coefficient of correlation was lower than in the younger than 50 , but still significant. In evaluating the utility of USQ as a screening for DEXA measurement, we found that $21.4 \%$ of patients with normal values for T score on USQ were osteopenic or osteporotic on TROC. This discrepancy was even more evident on LOMB, where $29.9 \%$ of the patients with normal DMO presented T<-1 on USQ, and $35.8 \%$ of the patients with normal USQ had $T<-1$ on DMO of the same region. The coefficient of variation of USQ obtained after 9 measurements in the same person on different days was $0.66 \%$ for SOS and $2.75 \%$ for BUA. In conclusion, the USQ is only moderately related to DMO and has little utility as a screening for DEXA examination. Its value to determine fracture risk is already established, however prospective studies are necessary to evaluate its usefulness in the diagnosis and follow-up of osteoporosis. (Arq Bras Endocrinol Metab 1999:43/3 205-209)

Keywords: Bone ultrasonometry; Bone densitometry; Bone mass

A MEDIÇÃO DA MASSA ÓSSEA através da densitometria óssea com raio X duo-energético (DEXA) é o parâmetro mais utilizado atualmentc para diagnóstico e acompanhamento da ostcoporose, e a grande maioria dos trabalhos da literatura mundial avaliando risco de fratura e resposta a esquemas terapêuticos utilizaram este método. A própria definição de osteoporose pela Organização Mundial de Saúde reunida cm Hong Kong cm 1994 estabeleceu critérios diagnósticos baseados em valores obtidos pela densitometria óssea, classificando como ostcopênicas aquelas mulheres que apresentarem-se entre 1 c 2,5 desviospadrão (DP) abaixo da média para adultas jovens e de osteoporóticas as que se cncontrarem com mais de 2,5 DP abaixo da média para adultas jovcns (1).

Embora discutiveis 110 que se refere a aspectos etiopatogênicos, estes dados têm sido de utilidade em estudos populacionais e na avaliação de risco de fratura de um indivíduo. Este método, devido a sua alta precisão e reprodutibilidade, foi difundido pelo mundo. Apresentam como desvantagens, no entanto, o fato de utilizarem uma fonte de radiação ionizante c possuírem um elevado custo que limita seu uso cm estudos populacionais, principalmente em nosso país, onde os recursos destinados à saúde da população mais carcnte são mínimos.

As grandes discussões mundiais em torno de contaminação ambiental e dos gastos com saúde estimulam a busca por métodos não poluentes e mais baratos, mas que tragam as mesmas informações que os métodos já estabelecidos. Em função disto, a ultrassonometria óssca quantitativa (USQ) vem sendo avaliada c alguns resultados promissores têm sido apresentados. Bascia-se na modificação das características de ultrassom transmitido através de ossos de localização mais superficiais, como no calcâneo, tíbia, falanges ou patela. Os sistemas atuais se constituem de dois transdutores, sendo um transmissor c outro receptor, posicionados em locais diferentes do osso a ser estudado. Os aparelhos existentes entre nós avaliam o calcâneo, osso predominantemente trabecular, através das medidas de velocidade de transmissão do ultrassom (SOS $\mathrm{cm} \mathrm{m} / \mathrm{s}$ ) e da atenuação da freqüiência do ultrassom (BUA cm dB/MHz).

A DEXA faz parte da rotina do clínico, que já possui intimidade suffciente com o método, conhecendo sua indicação e interpretando adequadamente seus resultados no diagnóstico c acompanhamento das doenças ósteo-metabólicas. A USQ vêm sendo apresentada aos médicos brasilciros pelos fabricantes destes aparelhos como um cxame que se prestaria ao rastreamento de mulheres nas quais a DEXA estaria indicada. Acreditamos que este cnfoque não scja verdadeiro, e por isso realizamos este trabalho.

Com a disponibilidade de utilização destes métodos de avaliação da massa óssea, fizemos uma comparação entre os resultados obtidos pelos dois métodos em pacientes rotineiramente encaminhados para realização de DMO no serviço de Endocrinologia da UNIFESP, e apresentamos nossos resultados.

\section{CASUÍSTICA E MÉTODO}

Foram avaliados 195 indivíduos com os mais variados diagnósticos encaminhados para o Serviço de Densitometria Óssea da Disciplina de Endocrinologia da UNIFESP. Destes, seis foram excluídos por apresentarem idade inferior a 20 anos. Dos 189 restantes, 165 eram mulheres e 24 homens, com idades entre 20 e 84 anos $\left(\mathrm{M}_{ \pm} \mathrm{DP}\right.$ : $51,8 \pm 13,1$; mediana de 51 anos para mulheres, e $51,7 \pm 18,5$; mediana de 53 anos para os homens). Todos foram submetidos, sem qualquer outro critério, a realizaÇão de DEXA (Lunar- DPX-L) cm região de coluna lombar (L2-L4) e proximal de fềmur (colo e trocânter) c USQ (Lmar-Aquilles) em calcâneo na mesma ocasiăo. A USQ foi realizada em pé direito, com a região plantar totalmente apoiada na superficic da cuba, que cra precnchida por água à temperatura de $37^{\circ} \mathrm{C}$ atć o nível do tornozclo, com paciente na posição sentada.

A USQ e a DEXA foram comparados em termos de DMO (em $\left.\mathrm{g} / \mathrm{cm}^{2}\right)$ em coluna lombar 
(LOMB), colo de fềmur (COLO) c trocanter maior (TROC) e USQ com os parâmetros de velocidade do som (SOS) e atcnuação do ultrassom (BUA) de calcâneo, e ainda em relação ao número de DPs distantes da média para a população de adultos jovens (T) $\mathrm{cm}$ ambos métodos (valores fornecidos pelo fabricante dos aparelhos). Para avaliação do coeficiente de variação $(\mathrm{CV})$ do método realizamos a medida de USQ em uma mesma pessoa por 9 vezes em ocasiões diferentes.

Os dados estão apresentados em média $\pm \mathrm{DP}$ e a correlação entre os variados parâmetros foi avaliada por regressão linear, considerando como significativo se $\mathrm{P}<0,05$.

\section{RESULTADOS}

Os valores obtidos para os diferentes parâmetros avaliados pela DEXA e USQ encontram-se na Tabela 1. Todos os parâmetros avaliados por ambos os métodos correlacionaram-se negativamente com a idade de maneira semelhante.

Observamos uma correlação positiva cstatisticamente significante entre as medidas de SOS c BUA com as medidas de DMO obtidas em todos os sítios avaliados pela DEXA, sendo que os melhores coeficientes de correlação foram obtidos entre SOS e TROC (Tabela 2).

Quando separamos os indivíduos por sexo, os homens apresentaram melhores correlações entre as medidas obtidas por USQ e por DEXA do que as mulheres (Tabcla 2). Da mesma forma, os indivíduos mais jovens (<50 anos) apresentaram correlações melhores entre os diversos parâmetros de USQ e DEXA, se comparados aos mais velhos (>50 anos) (Tabela 3 ).

Os índices $\mathrm{T}$ (número de DPs distantes da média de uma população de adultos jovens fornecidos pelo fabricante dos aparelhos) apresentaram em todos locais uma distribuição normal $\mathrm{cm}$ nossa população. No sentido de avaliarmos o valor da utilização da USQ como rastreamento para realização de DEXA, consideramos arbitrariamente o limite de até IDP abaixo da média como normal para ambos os métodos. Desta forma, pudemos notar uma grande discrepância entre os valores considerados normais ou alterados no mesmo indivíduo por estes dois diferentes métodos de avaliação da massa óssca.

Dentre os 89 pacientes considerados normais pela DEXA em coluna lombar, 29,8\% apresentavam valores abaixo de IDP na USQ. Por outro lado, $35,8 \%$ dos indivíduos cujos valores de USQ estavam normais (it menos de 1DP abaixo da média), apresentavam-se com osteopenia $(\mathrm{T}<-1)$ pela DEXA na mesma região (Tabela 4). A discordância foi pouco menor quando analisamos os parâmetros em trocânter, quando $21,4 \%$ dos indivíduos a mais de -IDP no USQ apresentavam-se osteopênicos ou ostcoporóticos pela DEXA (Figura l).

O CV dos valores obtidos de USQ para as 9 medidas realizadas na mesma pessoa em ocasiões diferentes foi de $0,66 \%$ para SOS e 2,75\% para BUA.

\section{DISCUSSĀO}

Nossos resultados são muito semclhantes aos já relatados nia literatura, onde os coeficientes de correlação entre DEXA c USQ variam de 0,34 a 0,72 (2). Coeficientes de correlação menores são observados quanto maior a homogeneidade da amostra, quando indivíduos são agrupados por idade ou sexo, ou por características clínicas $(3,4)$. Este fato também foi constatado por nós, quando limitamos a idade dos indivíduos acima de 50 anos, a população que mais se utilizaria destes métodos. Entretanto, uma excelente correlação foi observada no grupo dos homens, onde um $r=0,82$ foi cncontrado entre SOS c TROC. A reprodutibilidade do USQ também se mostra inferior à obtida pela DEXA na literatura, e talvez colabore de certa forma para a baixa correlação entre os métodos. Enquanto o CV da DMO normalmente localizase entre e 1 a $3 \%$, o mesmo parâmetro observado na ultrassonometria varia de 0,9 a $6,1 \%(5,6)$. Em nosso estudo obtivemos um CV de $2,75 \%$ para $B U A$ e $0,66 \%$ para SOS na USQ, e sabemos que a CV para os valores de DMO em nosso aparelho são de $0,9 \%$ para coluna e $1,5 \%$ para colo de fềmur (dados năo apresentados).

Tabela 1. Valores obtidos para as medidas de USQ e DEXA $(n=189)$ e seus respectivos coeficientes de correlação (CC) com a idade.

\begin{tabular}{lcccc}
\hline Parâmetro & M \pm DP & Medigna & CC (R) & P \\
SOS $(\mathrm{m} / \mathrm{s})$ & $1550 \pm 35,2$ & 1550 & 0,42 & $<0,0001$ \\
BUA $(\mathrm{dB} / \mathrm{MHz})$ & $115,0 \pm 12,5$ & 114 & 0,40 & $<0,0001$ \\
Coluna $\left(\mathrm{g} / \mathrm{cm}^{2}\right)$ & $1,080 \pm 0,18$ & 1,070 & 0,44 & $<0,0001$ \\
Colo de fêmur $\left(\mathrm{g} / \mathrm{cm}^{2}\right)$ & $0,871 \pm 0,169$ & 0,867 & 0,48 & $<0,0001$ \\
Trocânter maior $\left(\mathrm{g} / \mathrm{cm}^{2}\right)$ & $0,743 \pm 0,146$ & 0,730 & 0,40 & $<0,0001$ \\
\hline
\end{tabular}


Tabela 2. Coeficientes de correlação entre os parâmetros SOS e BUA obtidos na USQ e Os valores de DMO obtidos nos diferentes locais pela DEXA nos individuos separados por sexo.

\begin{tabular}{|c|c|c|c|c|c|c|}
\hline \multirow[b]{2}{*}{ DEXA } & \multicolumn{2}{|c|}{ Mulheres $(n=165)$} & \multicolumn{2}{|c|}{ Homens $(n=24)$} & \multicolumn{2}{|c|}{ Total $(n=189)$} \\
\hline & sos & BUA & sos & BUA & sos & BUA \\
\hline LOMB & 0,46 & 0.49 & 0,71 & 0,63 & 0,50 & 0,49 \\
\hline COLO & 0,49 & 0,55 & 0,77 & 0,72 & 0,54 & 0,56 \\
\hline TROC & 0,55 & 0.49 & 0,82 & 0,57 & 0,60 & 0.52 \\
\hline
\end{tabular}

Tabela 3. Coeficientes de correlação entre os parâmetros SOS e BUA obtidos na USQ e os valores de DMO obtidos nos diferentes locais pela DEXA separando o grupo de pacientes por idade.

\begin{tabular}{lllll}
\hline & \multicolumn{2}{c}{$>$ 50 anos } & \multicolumn{2}{c}{$<50$ anos } \\
\hline DEXA & SOS & BUA & SOS & BUA \\
LOMB & 0,31 & 0,42 & 0,47 & 0,40 \\
COLO & 0,39 & 0,42 & 0,48 & 0,49 \\
TROC & 0,42 & 0,38 & 0,60 & 0,52 \\
\hline
\end{tabular}

Tabela 4. Concordâncias e discordâncias entre os valores de T obtidos pelo USQ de calcâneo e DMO em coluna lombar.

\begin{tabular}{lrrl}
\hline Método & USQ T>-1 & USQ T<-1 & \multicolumn{1}{c}{ Total } \\
\hline DMO LOMB T>-1 & $61 \begin{array}{r}(68,5 \%) \\
(64,2 \%)\end{array}$ & $\begin{array}{r}28(31,5 \%) \\
(29,8 \%)\end{array}$ & $89(100 \%)$ \\
\hline DMO LOMB T<-1 & $34 \begin{array}{r}(34 \%) \\
(35,8 \%)\end{array}$ & $\begin{array}{r}66(66 \%) \\
(70,2 \%)\end{array}$ & $100(100 \%)$ \\
\hline Total & $95(100 \%)$ & $94(100 \%)$ & $189(106 \%)$ \\
\hline
\end{tabular}

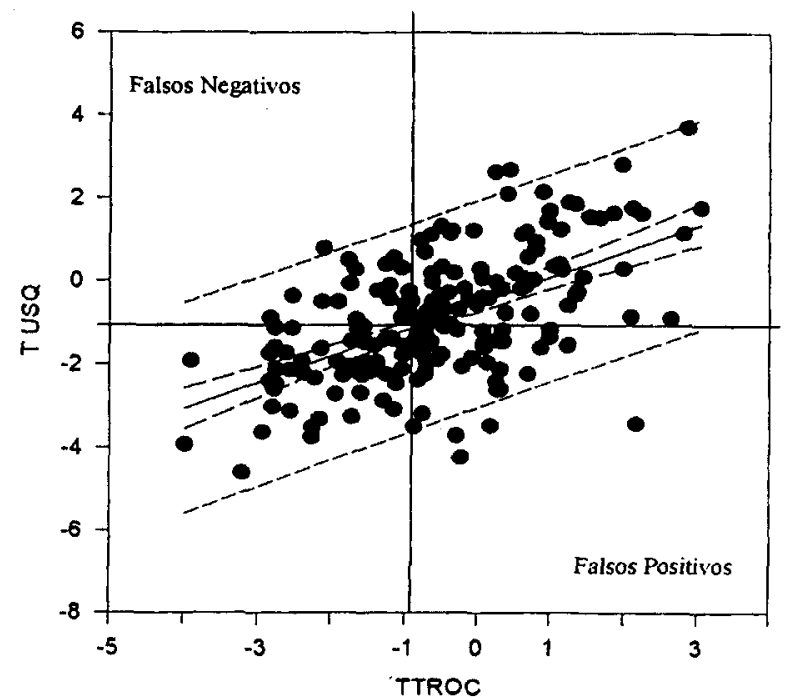

Figura 1. Regressão linear obtida para os valores de USQ de calcâneo e DMO em trocanter $(r=0,57, p<0,0001)$, representados pelo T (número de DPs distantes da média de uma população de adultos jovens), que resultou na expressão: T USQ $=-0,55+(0,63 \times$ T TROC). O quadrante superior esquerdo $(21,4 \%)$ representa os falsos negativos, isto é, aqueles individuos com USQ normal $(T>-1)$ porém com DEXA alterado $(T<-1)$.
A ultrassonometria se reduz ao longo da vida, de maneira semelhante a observada pela DMO, e também constatada por nós, através da correlação negativa obtida entre idade e os parâmetros de USQ. Estudos feitos com grupos de mulheres com e sem fraturas demonstram menores níveis de velocidade de transmissão do ultrassom (SOS) e da atenuação da freqüêencia do ultrassom (BUA) no segundo grupo (7). BUA tende a ser 15 a $30 \%$ inferior nestes casos quando comparados aos controles. Os níveis de BUA e SOS também são menores naquelas mulheres com fraturas de quadril, se comparadas àquelas com fraturas de vértebras ou rádio distal (8). Várias análises transversais e algumas longitudinais avaliaram risco de fraturas e medidas de massa óssea e constataram que USQ é tão ou até mais sensível para avaliar risco de fraturas do que DEXA $(9,10)$. Sabemos que a DEXA mede a quantidade de mineral (cálcio) no osso e que, em situações de osteopenia, este conteúdo está reduzido. Se ambos correlacionam-se de maneira independente com o risco de fratura, a apenas moderadamente entre si, a USQ deve medir uma característica óssea diferente da obtida pela DEXA. 
A DMO avaliada pela DEXA justifica cerca de 70 a $75 \%$ da variação da resistência óssea, enquanto que a porcentagem restante pode ser atribuída a fatores cumulativos e sinérgicos, como a microarquitetura óssea, a arquitetura, a presença de artefatos que interfiram na medida da DMO, além do estado de remodelação óssea (2). A grande vantagem atribuída ao USQ seria a sua capacidade em avaliar a microarquitetura do osso. Fatores locais, entretanto, também influenciam as medidas de USQ. Jones e col (11) demonstraram uma elevação dos níveis de ultrassonometria em mulheres jovens previamente sedentárias após um ano de atividade fisica programada. Além disto, as diferenças significativas encontradas entre nas medições de US de calcâneo nos lados dominante e não dominante principalmente entre homens jovens reforça este conceito (5).

Concluindo, a USQ correlaciona-se apenas moderadamente com DMO e, portanto, sua utilização como um teste de rastreamento para a realização de DEXA não tem utilidade, uma vez que a discordância entre os métodos para um mesmo indivíduo é clevada. O valor da USQ na avaliação do risco de fratura já está estabelecido, porém estudos prospectivos são necessários para que se padronize sua utilidade no diagnóstico e acompanhamento das doenças ósseas.

\section{REFERÊNCIAS}

1. World Health Organization. Assessment of fracture risk and its application to screening for postmenopausal osteoporosis. Technical report series 843, 1999. Geneva:WHO.

2. Njeh CF, Boivin CM. Langton CM. The role of ultrasound in the assessment of osteoporosis: a review. Osteoporosis Int 1997;7:7-22.

3. Baran DT, Kelly AM, Karellas A, et al. Ultrasound attenuation of the calcis in women with osteoporosis and hip fractures, Calcif Tissue Int 1988;43:138-42.
4. Massie A, Reid DM, Porter RW. Screening for osteoporosis: comparison between dual energy $X$-ray absortiometry and broad band ultrasound attenuation in 1000 perimenopausal women. Osteoporosis Int 1993;3:107-10.

5. Howard GM, Nguyen TV, Pocock NA, Kelly PJ, Eisman JA. Influence of handedness on calcaneal ultrasound implications for assessment of osteoporosis and study design. Osteoporosis Int 1997;7:190-4.

6. Rosenthall L. Influence of bone quality on precision of calcaneal ultrasonometry. Calcif Tissue Int 1997:61:139-41.

7. Hans D, Dargent-Molina $P$, Schott AM, et al. Ultrasonographic heel measurements to predict hip fracture in elderly women: the EPIDOS prospective study. Lancet 1996;348:51 1-4.

8. Agren M, Karellas A, Leahy D, Marks S, Baran D. Ultrasound attenuation of the calcaneus: a sensitive and specific discriminator of osteopenia in postmenopausal women. Calcif Tissues Inf 1991;48:240-4

9. Turner CH, Peacock M, Timmerman L, Neal JM, Johnston CC. Calcaneal ultrasonic measurement discriminate hip fracture independently of bone mass. Osteoporosis Int 1995:5: 130-5

10. Bauer DC, Glüer CC, Cauley JA, et al. Bone ultrasound predicts fracture strongly and independently of densitometry in older women: a prospective study. Arch Intern Med 1997; 157:629-34

11. Jones PR, Hardman AE, Hudson A, Norgan NG. Influence of brisk walking on the broadband ultrasonic attenua tion of the calcaneus in previosly sedentary women aged 30-61 years. Calcif Tissue Int 1991:49:112-5.

\section{Endereço para correspondência:}

Marise Lazaretti Castro

Disciplina de Endocrinologia

UNIFESP / EPM

Caixa Postal 20.266

04034-970 São Paulo, SP 